\title{
REDUÇÃO DE APLICAÇÕES DE INSETICIDAS ATRAVÉS DA ADOÇÃO DE TÁTICAS DE MANEJO INTEGRADO DO Triozoida limbata (ENDERLEIN, 1918) (HEMIPTERA: TRIOZIDAE) EM GOIABEIRA ${ }^{1}$
}

\author{
WILSON CARLOS PAZINI ${ }^{2} \&$ JÚLIO CESAR GALLI ${ }^{3}$
}

RESUMO- A praga de goiabeira que tem requerido maior frequência de controle, na região de JaboticabalSP, nos últimos anos, é o psilídeo Triozoida limbata. Assim, esta pesquisa objetivou: a) Comparar táticas de controle de T. limbata baseadas no monitoramento e na seletividade de inseticidas, visando a diminuir as aplicações; b) Registrar e correlacionar os inimigos naturais habitantes no agroecossistema goiabeira com a praga-chave e com fatores meteorológicos; c) Constatar se o controle de T. limbata afeta a população de moscas-das-frutas, e d) Verificar a eficiência de inseticidas adequados ao MIP, no controle de T. limbata. O experimento foi conduzido com a cultivar Paluma, em 2004, no município de Vista Alegre do Alto-SP. As estratégias utilizadas foram (doses em g.i.a./100L de água): testemunha; imidacloprid $(4,0)$; imidacloprid + beta-cyfluthrin $(2,5+0,3)$; acetamiprid $(4,0)$; fenpropathrin $(15,0)$, e tratamento convencional regional. Através dos resultados, conclui-se que é possível diminuir o número de aplicações e utilizar inseticidas menos agressivos ao meio ambiente e ao homem, com a adoção do monitoramento de T. limbata e aplicação no nível de ação. As densidades populacionais dos inimigos naturais (Scymnus spp., Cycloneda sanguinea, Azia luteipes, Crysoperla spp., Polybia spp., Brachygastra spp.) apresentam correlações positivas com as densidades populacionais de T. limbata. As flutuações populacionais de T. limbata e dos inimigos naturais não são alteradas pelos fatores meteorológicos (precipitação e temperatura), em pomar irrigado. A população de Anastrepha spp. é minimizada quando as aplicações são para controlar T. limbata. Os inseticidas imidacloprid, imidacloprid + beta-cyfluthrin, acetamiprid e fenpropathrin são eficientes no controle de T. limbata.

Termos para indexação: Insecta, Coccinelidae, Triozidae, armadilhas, Psidium guajava.

\section{REDUCTION OF INSECTICIDES APPLICATIONS THROUGH THE ADOPTION OF INTEGRATED MANAGEMENT TACTICS OF Triozoida limbata (ENDERLEIN, 1918) (HEMIPTERA: TRIOZIDAE) IN GUAVA TREE}

ABSTRACT- The guava tree pest that has been requesting larger control frequency, in the area of Jaboticabal - São Paulo in the last years, is the psilídeo Triozoida limbata. Thus, the objectives of this research were: a) Compare control tactics $T$. limbata based on monitoring and selectivity of insecticide to decrease the applications; b) To register and to correlate the natural enemies in habitants in guava tree ecosystem with the key pest and with meteorological factors; c) See if the control of T. limbata affects the population of fruit flies and d) To verify the efficiency of appropriate insecticides to IMP, in control of T. limbata. The experiment was conducted in 2004, in an orchard of Paluma cultivar in Vista Alegre do Alto, SP. The used strategies were (doses in g.a.i./100L water): control; imidacloprid + beta-cyfluthrin $(2.5+0.3)$; acetamiprid (4.0); fenpropathrin (15.0) and regional conventional treatment. It was concluding that is possible to reduce the number of applications of less aggressive insecticides to the environment and the man, with the adoption of monitoring of T. limbata and implementing the action level. The natural enemies' populations densities (Scymnus spp., Cycloneda sanguinea, Azia luteipes, Crysoperla spp., Polybia spp., Brachygastra ssp.) present positive correlations with the populations of T. limbata. Population fluctuations of T. limbata and natural enemies are not altered by meteorological factors (precipitation and temperature) in irrigated orchard. The population of Anastrepha spp. is minimized when of the applications is for control T. limbata. The insecticides imidacloprid, imidacloprid + beta-cyfluthrin, acetamiprid and fenpropathrin are efficient in the control of T. limbata.

Index terms: Insecta, Coccinelidae, Triozidae, traps, Psidium guajava.

\footnotetext{
'(Trabalho 076-10). Recebido em: Parte da tese de doutorado do primeiro autor na UNESP/FCAV em Jaboticabal-SP.

${ }^{2}$ Engenheiro Agrônomo - Doutor em Entomologia Agrícola - Faculdade de Ciências Agrárias e Veterinárias, Campus de Jaboticabal, Unesp - Univ Estadual Paulista, Via de Acesso Prof. Paulo Donato Castellane s/n CEP 14.884-900 Jaboticabal - SP, Brasil e-mail: wpazini@fcav.unesp.br

${ }^{3}$ Professor Doutor - Faculdade de Ciências Agrárias e Veterinárias, Câmpus de Jaboticabal, Unesp - Univ Estadual Paulista, Via de Acesso Prof. Paulo Donato Castellane s/n CEP 14.884-900 Jaboticabal - SP, Brasil e-mail: jcgalli@fcav.unesp.br
} 


\section{INTRODUÇÃO}

A região agrícola de Jaboticabal-SP, destaca-se por ser a maior produtora de goiaba do Estado de São Paulo. A praga que tem requerido mais aplicações de agrotóxicos nessa região é o psilídeo Triozoida limbata (Enderlein, 1918) (Hemiptera: Triozidae), seguido pela moscas-dasfrutas Anastrepha spp. (Diptera: Tephritidae), o besouro-amarelo Costalimaita ferruginea (Fabr., 1801) (Coleoptera: Chrysomelidae), o gorgulhoda-goiabeira Conotrachelus psidii (Marshall, 1922) (Coleoptera: Curculionidae) e o percevejo Leptoglossus gonagra (Fabr., 1775) (Hemiptera: Coreidae).

As joaninhas da família Coccinellidae (Coleoptera) são, reconhecidamente, importantes inimigos naturais de psilídeos (MICHAUD, 2001). $\mathrm{Na}$ cultura da goiaba, Barbosa et al. (1999) citaram como realizadores de controle biológico do Triozoida sp.: joaninhas, aracnídeos, crisopídeos, sinferobídeos, sirfídeos, tacnídeos, estafilinídeos, nabídeos, moscas cecidomídeas, microhimenópteros calcidídeos e encirtídeos e Cladosporium cladosporioides.

Entretanto, o controle biológico e as medidas culturais não conseguem manter o psilídeo da goiabeira em baixas infestações. Assim, agricultores desta região passaram a utilizar os inseticidas fenitrothion (Sumithion 500 EC), fenthion (Lebaycid 500), dimethoate (Dimetoato CE), methamidophos (Hamidop 600), lambda-cyhalothrin (Karate Zeon 250 CS), carbosulfan (Marshal 200 SC) e acephate (Orthene 750 BR) no controle do psilídeo. Entretanto, estes inseticidas não possuem registro no Ministério da Agricultura Pecuária e Abastecimento, para esta praga. $\mathrm{O}$ único inseticida registrado para controle de T. libata é o imidacloprid (Provado 200 SC).

O conhecimento do agroecossistema da goiabeira, como uma unidade ecológica complexa, possibilita o aperfeiçoamento de programas de Manejo Integrado de Pragas (MIP). Para isso, as pragas e os inimigos naturais devem ser monitorados diretamente nas folhas e nos frutos ou por meio de armadilhas (PARAJULEE; SLOSSER, 2003).

Diante do exposto, o presente tabalho teve como objetivos: a) Comparar táticas de controle de $T$. limbata baseadas no monitoramento populoacional e na seletividade de inseticidas, visando a diminuir as aplicações; b) Registrar e correlacionar os inimigos naturais habitantes no agroecossistema goiabeira com a praga-chave e com fatores meteorológicos; c) Constatar se o controle de T. limbata afeta a população de moscas-das-frutas, e d) Verificar a eficiência de inseticidas adequados ao MIP, no controle de T. limbata.

\section{MATERIAL E MÉTODOS}

O experimento foi realizado em um pomar de goiaba na safra agrícola de 2004, em Vista Alegre do Alto-SP, com a cultivar Paluma, plantada em outubro de 1995 . O cultivo foi conduzido com poda drástica, realizada em 15-11-2003. As irrigações foram realizadas pelo sistema de aspersão com canhão autopropelido. A adubação e o controle de plantas daninhas foram realizados conforme o padrão usual da região.

As estratégias utilizadas foram (doses em g.i.a./100L de água): 1) Testemunha: sem aplicação de inseticida; 2) Estratégia imidacloprid 4,0 (Provado 200 SC); 3) Estratégia imidacloprid + beta-cyfluthrin 2,5 + 0,3 (Connect); 4) Estratégia acetamiprid 4,0 (Mospilan); 5) Estratégia fenpropathrin 15,0 (Danimen 300 EC); 6) Tratamento convencional regional (inseticidas normalmente utilizados pelos produtores): dimethoate 40,0 (Dimetoato CE), methamidophos (Hamidop 600) + óleo mineral (Assist) 30,0 + 242,5, lambda-cyhalothrin 1,25 (Karate Zeon $250 \mathrm{CS}$ ), carbosulfan 10,0 (Marshal $200 \mathrm{SC}$ ) e acephate 37,5 (Orthene $750 \mathrm{BR}$ ).

O delineamento estatístico utilizado foi o de blocos casualizados, com as seis estratégias repetidas quatro vezes. Cada parcela experimental foi constituída por três plantas, sendo as amostragens realizadas na planta central. As aplicações dos inseticidas foram realizadas com um pulverizador turboatomizador tratorizado de arrasto, fechandose um lado do equipamento. Assim, as aplicações foram feitas dos dois lados da planta, devido ao grande enfolhamento e à dificuldade de as gotas atingirem o centro da planta adulta. $\mathrm{O}$ volume de calda pulverizado na planta foi de dez litros (cinco de cada lado).

Os inseticidas foram aplicados somente quando as infestações médias atingiram $30 \%$ das folhas com a presença de ninfas do psilídeo, nas estratégias de MIP. Este nível de ação está em conformidade com o preconizado pela Produção Integrada de Goiaba (adaptado de JACOMINO et al., 2002).

O monitoramento de T. limbata foi feito através da coleta semanal de dez folhas novas (do primeiro ou do segundo par do ramo) por parcela, totalizando 40 folhas por estratégia. Estas folhas foram acondicionadas em sacos de papel etiquetados e conduzidos ao Laboratório de Seletividade Ecológica da UNESP/FCAV Câmpus de Jaboticabal-SP, onde, com o auxílio de microscópio 
estereoscópio, registrou-se o número de ninfas vivas por folha.

As moscas-das-frutas foram monitoradas através da utilização de frascos caça-moscas confeccionados partindo-se de frascos de álcool com capacidade de um litro (adaptado de VELOSO et al., 1994). A isca utilizada foi proteína hidrolisada de milho, produto comercial Moscatex, na concentração de $3 \%$. Foram colocados $250 \mathrm{~mL}$ desta calda dentro de cada frasco caça-moscas e instalados a um metro e meio do solo, na planta central de cada parcela. Semanalmente, os frascos caça-moscas eram substituídos e avaliados no laboratório, onde se fazia a triagem dos insetos capturados. Assim, montou-se uma coleção entomológica, registrando os insetos por parcela e por data de amostragem.

Armadilhas adesivas amarelas foram utilizadas para monitorar os inimigos naturais (WALTON; PRINGLE, 2004; ALBAJES et al., 2009) e também as moscas-das-frutas (ROBACKER; RODRIGUEZ, 2004) por interceptação de voo e atratividade da cor. Essas armadilhas apresentavam as medidas de 25 centímetros de comprimento por 9,5 centímetros de largura e cola nas duas faces, que servem para capturar os insetos. Foi colocada uma armadilha a 1,5 metro do solo, no interior da planta central de cada parcela, totalizando 24 armadilhas no experimento. As armadilhas foram mantidas no campo por 15 dias e imediatamente substituídas por novas, sem interrupção, sendo acondicionadas em pastas de plástico e conduzidas ao labotatório para avaliação. Com os insetos capturados, foi confeccionada uma coleção entomológica, para identificação e registro.

Para fins de análise estatística, os números de ninfas de psilídeos por folha foram submetidos ao teste F, para análise de variância, e ao teste de Tukey, para comparação das médias. As percentagens de redução das pragas foram calculadas pela fórmula de Abbott (1925). Para a obtenção das correlações estatísticas, os dados obtidos foram submetidos à análise de correlação linear simples.

\section{RESULTADOS E DISCUSSÃO}

A infestação do psilídio encontrava-se abaixo de $30 \%$ de folhas novas atacadas em todos os tratamentos, no início da safra (Figura 1). Mesmo assim, o agricultor realizou uma pulverização com o inseticida dimethoate, na dose de 40,0 g.i.a./100L de água, em 13-02-04, visando ao controle preventivo do T. limbata.

Todas as estratégias apresentaram acentuada elevação dos números de ninfas de T. limbata (Tabela
1) e também das percentagens de folhas atacas (Figura 1), no período de 27-02-04 a 04-03-04, superando $30 \%$ de infestação. Esse aumento populacional determinou a primeira aplicação, que foi realizada no dia 08-03-04. Essa intervenção foi adaptada de Jacomino et al. (2002), que estabeleceram o nível de ação de $30 \%$ das plantas infestadas. A exceção foi no tratamento convencional, onde o agricultor realizou aplicação, no dia 04-03-04, com os inseticidas methamidophos, na dose de 30,0 g.i.a./100L de água + óleo mineral na dose de 242,5 g.i.a./100L de água.

A rapidez no aumento populacional do psilídeo foi verificado por nova amostragem realizada em 08-03-04, antes da aplicação dos inseticidas. Observou-se que as percentagens médias que se encontravam entre 30 e 50\%, no dia 27-02-04, subiram para 60 a 80\%, no dia 08-03-04 (Figura 1). A maior quantidade de ninfas em todas as estratégias, que no dia 27-02-04 era de 75,3 ninfas em dez folhas, passou para 234,3 no dia 08-03-04 (Tabela 1).

As percentagens de folhas atacadas permaneceram altas na testemunha, no período de 15 a 30-03-04, de 07 a 22 dias após a primeira aplicação (D1 ${ }^{\mathrm{a}}$ ) (Figura 1) (Tabela 1). Houve redução das percentagens de folhas atacadas nas estratégias de MIP, que diferiram significativamente da testemunha no número médio de ninfas de $T$. limbata (Tabela 1). As percentagens de eficiências, calculadas pela fórmula de Abbott (1925), foram altas em todas as estratégias de MIP (Figura 2). A eficiência do inseticida imidacloprid 200 CS (2 mL i.a./ planta), com a aplicação do produto puro nos ramos que saíam do tronco, no controle de Triozoida sp., também foi constatada por Barbosa et al. (1999), em experimento realizado no município de Petrolina-PE, que o citaram como seletivo aos inimigos naturais.

A infestação de T. limbata nas folhas novas começou a aumentar a partir de 13-04-04 (Figura 1), não apresentando diferenças significativas entre as estratégias (Tabela 1). Mesmo assim, o agricultor pulverizou acephate na dose de $37,5 \mathrm{~g}$ i.a./hL, em $26-$ 03-04 e lambda-cyhalothrin na dose de 1,25 g.i.a./100L de água, em 14-04-04, no tratamento convencional. Nas amostragens seguintes, dos dias 20-04-04 e 26-04-04, as estratégias atingiram o nível de ação de $30 \%$ ou mais de folhas atacadas pelo psilídeo, determinando a segunda intervenção para redução populacional, realizada em 26-04-04 (Figura 1).

No período de 04-05-04 a 18-05-04, de 08 a 22 dias após a segunda aplicação (D2 ${ }^{\mathrm{a}}$ ), todas as estratégias diferiram significativamente da testemunha no número médio de ninfas de T. limbata (Tabela 1). As percentagens de eficiência foram 
altas em todas as estratégias de MIP (Figura 2). No tratamento convencional, o agricultor pulverizou carbosulfan na dose de 10,0 g.i.a./100L de água, em 04-05-04.

As percentagens médias de folhas atacadas permaneceram baixas nos tratamentos, com exceção da testemunha, no período de 25-05-04 a 07-06-04 (Figura 1). Apesar disso, o agricultor realizou mais uma pulverização no tratamento convencional com inseticida acephate, na dose de 37,5 g.i.a./100L de água, em 26-05-04. Entretanto, uma infestação generalizada de lagartas geometrídeas induziu o agricultor a pulverizar toda a área de pesquisa com o inseticida lambda-cyhalothrin, na dose de 1,25 g.i.a./100L de água, em 11-06-04, que reduziu as percentagens de infestação do psilídeo observadas no dia16-06-04, principalmente na testemunha (Tabela 1).

A flutuação populacional de T. limbata, obtida pela média do número de psilídeo na testemunha, não foi alterada pelos fatores meteorológicos, pois não ocorreram correlações lineares significativas (precipitação acumulada semanal $\mathrm{r}=0,1328^{\text {ns }}$, temperatura mínima semanal $\mathrm{r}=0,3141^{\mathrm{ns}}$ e temperatura média semanal $\left.r=0,1945^{\text {ns }}\right)$. Estes resultados diferem dos relatados por Dalberto et al. (2004), que observaram que altas temperaturas são favoráveis ao aumento das populações de T. limbata em plantas de goiabeiras, na região de Londrina-PR, e que as menores populações de $T$. limbata ocorreram na estação seca, com menor temperatura e pluviosidade. Diferiram também dos resultados encontrados por Colombi e Galli (2009), que avaliaram o número de ninfas e a percentagem de dano causado nas folhas por T. limbata. Analisaram estes parâmetros correlacionados aos fatores meteorológicos. Concluíram que a densidade populacional do psilídeo aumentou com o surgimento das brotações e apresentou uma correlação positiva com a temperatura. Uma explicação para as diferenças pode estar relacionada à condução do pomar por irrigação, que ocasiona sucessivas brotações na planta. Mas novos estudos devem ser realizados em pomares irrigados.

Nas avaliações dos 408 frascos caçamoscas instalados, constatou-se uma Anastrepha spp. no dia 15-03-04 na estratégia imidacloprid + betacyflutrin e outra no tratamento convencional. As 192 armadilhas adesivas amarelas capturaram apenas duas moscas-das-frutas, sendo a primeira no dia 25-05-04 na testemunha e a segunda no dia 2206-04 na estratégia fenpropathrin. Esta baixíssima infestação de moscas-das-frutas ocorreu devido ao elevado número de aplicações realizadas para controle do psilídeo, principalmente no tratamento convencional regional.

A testemunha apresentou, no geral, as maiores quantidades de inimigos naturais, no período em que os inseticidas foram aplicados. Entre os inimigos naturais destacaram-se Scymnus spp. (Coleoptera: Coccinellidae) Cycloneda sanguinea (Linnaeus, 1763) (Coleoptera: Coccinellidae), Azia luteipes (Mulsant, 1850) (Coleoptera: Coccinellidae), Crysoperla spp. (Neuroptera: Chrysopidae), Polibia spp. (Hymenoptera: Vespidae) Brachygastyra spp. (Hymenoptera: Vespidae) pela abundância e frequência (Figura 3). Estes resultados assemelham-se aos encontrados por Barbosa et al. (2003), que constataram a presença dos coccinelídeos $C$. sanguinea, Eriopis conexa (Germar, 1824) (Coleoptera: Coccinellidae) e Scymnus sp., crisopídeos, aracnídeos, sirfídeos, nabídeos e tacnídeos nas goiabeiras do Nordeste brasileiro. Também, Takemoto et al. (1998) verificaram que o baixo período residual do imidacloprid resultou em pouco efeito adverso na colonização e na reprodução das espécies de Orius spp. Assim, consideraram que o imidacloprid pode ser compatível com programas de MIP. A utilização dos inseticidas acetamiprid e o imidacloprid em programas de MIP, em diversas culturas, corroboram os resultados encontrados por Ishaaya et al. (2001). A estratégia convencional apresentou, no geral, a menor quantidade de inimigos naturais (Figura 3).

As densidades populacionais dos inimigos naturais, anteriormente citados, demonstraram associação com a densidade populacional do $T$. limbata na testemunha, pois apresentaram correlações lineares simples significativas positivas $(\mathrm{r}=$ $0,5462 *$ ). As flutuações populacionais dos inimigos naturais, obtidas pela somatória de espécie anteriormente citada na testemunha, não foram alteradas pelos fatores meteorológicos, pois não ocorreram correlações lineares significativas (precipitação acumulada semanal $\mathrm{r}=0,2471^{\mathrm{ns}}$, temperatura mínima semanal $r=0,1100^{\text {ns }}$ e temperatura média semanal $\left.\mathrm{r}=0,2555^{\text {ns }}\right)$. $O$ pico populacional dos inimigos naturais ocorreu no dia 10-05-04 com 23 inimigos naturais capturados durante 14 dias, nas quatro armadilhas adesivas amarelas da testemunha (Figura 3). 


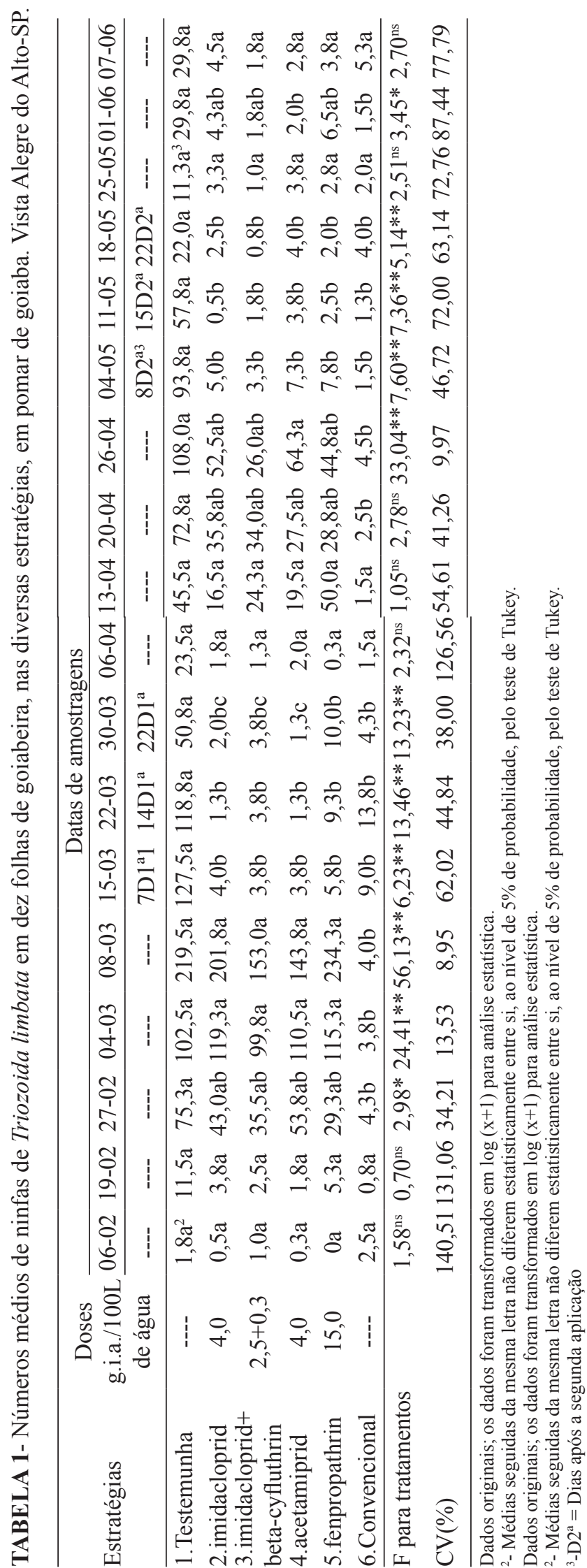




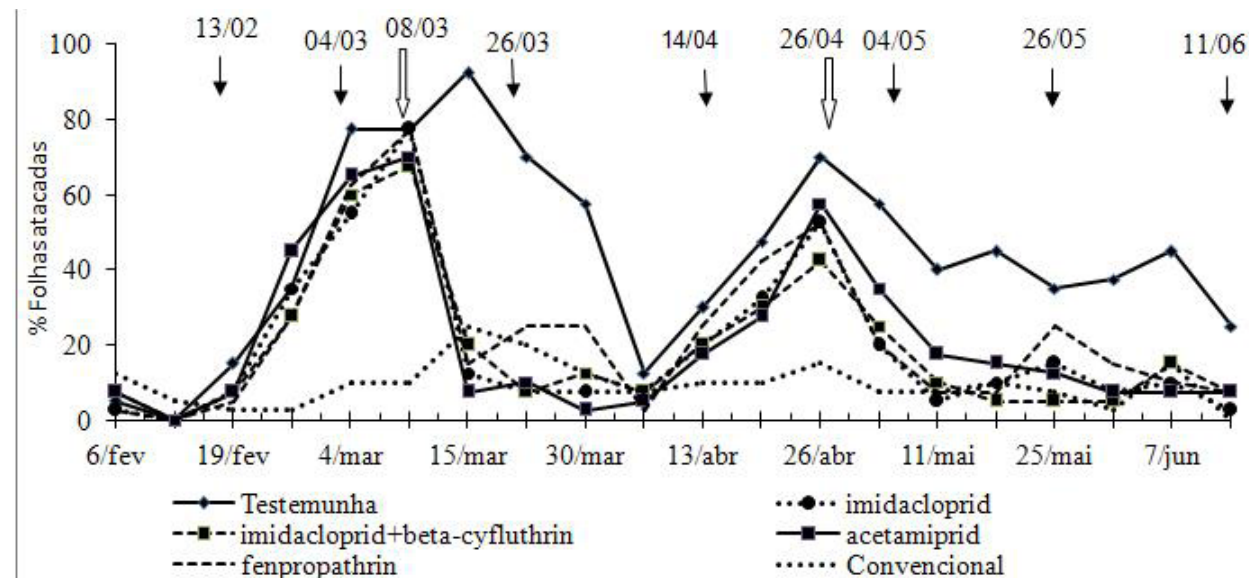

FIGURA 1 - Percentagens médias de folhas atacadas por Triozoida limbata e aplicações dos inseticidas realizadas nas estratégias, em pomar de goiaba. Vista Alegre do Alto-SP.

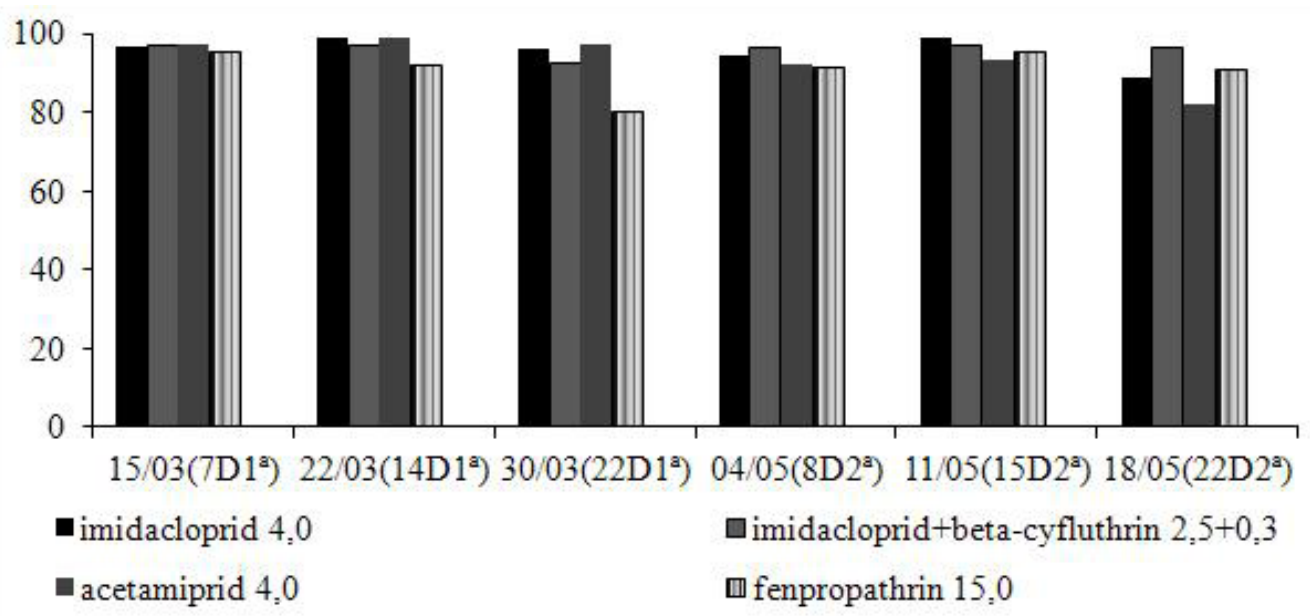

FIGURA 2 - Percentagens de eficiências, calculadas após as aplicações dos inseticidas, nas diversas estratégias de controle Triozoida limbata, em pomar de goiaba (D1 ${ }^{\mathrm{a}}=$ após a primeira aplicação; D2 ${ }^{\mathrm{a}}=$ Dias após a segunda aplicação). Vista Alegre do Alto-SP.

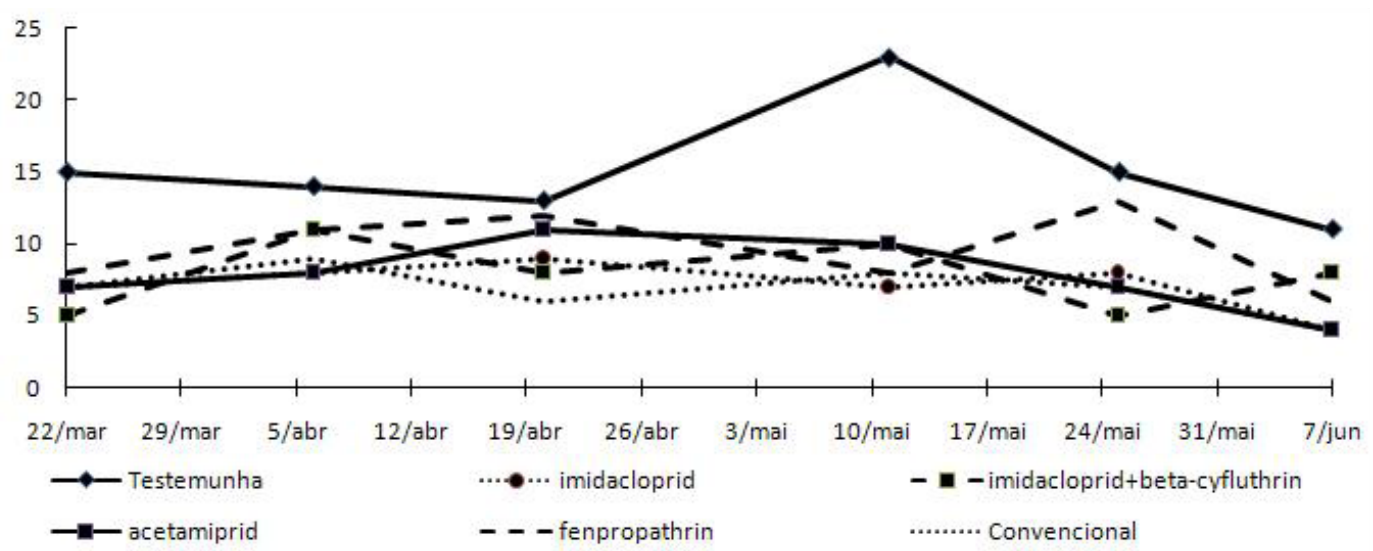

FIGURA 3 - Flutuações populacionais de inimigos naturais nas quatro armadilhas adesivas amarelas, por estratégia, em pomar de goiaba. Vista Alegre do Alto-SP. 


\section{CONCLUSÕES}

1-É possível diminuir o número de aplicações e utilizar inseticidas menos agressivos ao meio ambiente e ao homem, com a adoção do monitoramento de Triozoida limbata, e aplicação no nível de ação.

2-As densidades populacionais dos inimigos naturais (Scymnus spp., Cycloneda sanguinea, Azia luteipes, Crysoperla spp., Polybia spp. e Brachygastra spp.) apresentam correlações positivas com as densidades populacionais de Triozoida limbata. As flutuações populacionais de T. limbata e dos inimigos naturais não são alteradas pelos fatores meteorológicos (precipitação e temperatura), em pomar irrigado.

3-A população de Anastrepha spp. é minimizada quando as aplicações de inseticidas são para controlar TTriozoida limbata.

4-Os inseticidas imidacloprid, imidacloprid + beta-cyfluthrin, acetamiprid e fenpropathrin são eficientes no controle de Triozoida limbata.

\section{REFERÊNCIAS}

ABBOTT, W. S. A method of computing the effectiveness of an insecticide. Journal of Economic Entomology, Maryland, v. 18, n. 1, p. 265-267, 1925.

ALBAJES, R.; LUMBIERRES, B.; PONS, X. Responsiveness of arthropod herbivores and their natural enemies to modified weed management in corn. Environmental Entomology, State College, v. 38, n. 3, p. 944-954, 2009.

BARBOSA, F. R.; FERREIRA, R. G.; KIILL, L. H. P.; SOUZA, E. A.; MOREIRA, W. A.; ALENCAR, J. A. A.; HAJI, F. N. P. Nível de dano, plantas invasoras hospedeiras, inimigos naturais e controle do psilídeo da goiabeira (Triozoida sp.) no submédio São Francisco. Revista Brasileira de Fruticultura, Jaboticabal, v. 25, n. 3, p. 425-428, 2003.

BARBOSA, F. R.; SANTOS, A. P.; HAJI, A. T.; MOREIRA, W. A.; HAJI, F. N. P.; ALENCAR, J. A. Eficiência e seletividade do imidacloprid e lambdacyhalothrin no controle do psilídeo (Triozoida sp.), em goiabeira. Revista Brasileira de Fruticultura, Jaboticabal, v. 21, n. 3, p. 385$387,1999$.

COLOMBI, C. A.; GALLI, J. C. Dinâmica populacional e evolução de dano de Triozoida limbata
(Hemiptera: Psillydae) em goiabeira, em JaboticabalSP. Ciência e Agrotecnologia, Lavras, v. 33, n. 2 , p. 412-416, 2009.

DALBERTO, F. M. S.; MENEZES JUNIOR, A O.; SIMÕES, H. C.; BENITO, N. P.; PITWAK, J. Flutuação populacional do psilídeo-da-goiabeira, Triozoida limbata (Hemiptera: Psillydae) na região de Londrina-PR. Semina: Ciências Agrárias, Londrina, v. 25, n. 2, p. 87-92, 2004.

ISHAAYA, I.; KONTSEDALOV, S.; MAZIROV, D.; HOROWITZ, A. R. Biorational agents: Mechanism and importance in IPM and IRM programs for controlling agricultural pests. Mededelingen Faculteit Landbouwkundige en Toegepaste Biologische Wetenschappen Universiteit Gent, Dagan, v. 66, n. 2, p. 363-374, 2001.

JACOMINO, A. P.; PIZA Jr., C. T.; MURAKAMI, J. Y.; BALDIN, J. C.; FIORANI, L. A.; KUMAGAI, L. Y.; CONTI, L. H.; OLIVEIRA, M. E. R.; MORI, M.; SOUZA FILHO, M. F.; KAVATI, R.; ARAÚJO, V. B. Manejo integrado de pragas na cultura da goiaba. Campinas: CATI, 2002. 11p. Apostila $3^{\mathrm{a}}$ versão

MICHAUD, J. P. Numerical response of Olla vnigrum (Coleoptera: Coccinellidae) to infestations of Asian citrus psyllid, (Hemiptera: Psyllidae) in Florida. Florida Entomology, Lake Alfred, v. 84, n. 4, p. 608-612, 2001.

PARAJULEE, M. N.; SLOSSER, J. E. Potential of yellow sticky traps for lady beetle survey in cotton. Journal of Economic Entomology, Fresno, v. 96, n. 1, p. 239-245, 2003.

ROBACKER, D. C.; RODRIGUEZ, M. E. A simple and effective cylindrical sticky trap for fruit flies (Diptera: Tephritidae). Florida Entomologist, Lake Alfred, v.87, n. 4, p. 492-495, 2004.

TAKEMOTO, H.; OHNO, K.; MASUNAGA, T. Effective use of imidacloprid granules as a compatible chemical with integrated pest management programs in eggplant [Solanum melongena] feilds. Bulletin Fukuoka Agricultural Ressearch Center, Chikushino, v. 16, n. 17, p. 97-101, 1998.

VELOSO, V. R. S.; FERNANDES, P. M.; ROCHA, M. R.; QUEIROZ, M. V.; SILVA, R. M. R. Armadilha para monitoramento e controle das moscas-das-frutas Anastrepha spp. e Ceratitis capitata (Wied.) Anais da Sociedade Entomológica do Brasil, Londrina, v. 23, n. 3, p. 487-493, 1994.

WALTON, V. W.; PRINGLE, K. L. A survey of mealy bugs and associated natural enemies in vineyards in the Western Cape province, South Africa. South African Journal f Enology and Viticulture, Stellenbosch, v. 25, n.1, p. 23-25, 2004. 